\title{
Injecting a Liquid in Weightlessness: Droplet or Geyser Formation
}

\author{
Steven H. Collicott ${ }^{1}$ and Kyle D. Kennedy ${ }^{2}$ \\ ${ }^{1}$ School of Aeronautics and Astronautics, Purdue University, West Lafayette, IN; ${ }^{2}$ Electroimpact, \\ Mukilteo, WA
}

\begin{abstract}
Injecting a liquid into a gas-filled vessel while in weightlessness can result in at least two conditions - a droplet attached to the wall around the injection orifice and a geyser in which the liquid propagates away from the orifice in a continuous jet. The need to design injection of liquid to accomplish one condition or the other shows up in both zero-g fluids research geometries and spaceflight systems. Previous experiments by others assumed the rim of the injection orifice to be sharp. Liquid flow out of orifices with chamfered and rounded rims during the weightlessness of parabolic aircraft flight are studied in this work. When compared to previous work, results indicate that chamfered and rounded rims have little effect on the value of Weber number dividing the wall-bound droplet and geyser behaviors. Because any manufactured orifice will have finite bluntness, this conclusion is useful for both research and spaceflight systems.
\end{abstract}

Key words: $\quad$ Droplet Formation; Liquid Injection; Fluid Dynamics

Correspondence to: Steven H. Collicott School of Aeronautics and Astronautics Purdue University

701 W. Stadium Ave.

West Lafayette, IN 47907-2045

Telephone: 765-494-2339

E-mail: collicott@purdue.edu

\section{INTRODUCTION}

Several drop tower experiments in the first two decades of U.S. spaceflight studied the initial flow of a liquid injected into a gas-filled vessel. It was recognized early (Symons et al., 1968) that in the absence of gravity, the relative magnitudes of the momentum of the liquid flow and the capillary pressure difference across the free surface, described now as a Weber number, determine whether the liquid forms a droplet attached to the wall or if the liquid travels out into the vessel as a "geyser." The droplet and geyser conditions are sketched in Figure 1 and sample images from flight-test videos are presented in Figure 2. The droplet is the lower momentum result and the geyser the higher momentum result. The division between these two results is of interest. Presumably, a significant additional increase in liquid momentum could result in a spray of the liquid but such high speeds are not of interest here.

Early work was apparently motivated by spacecraft systems, with liquid injected into vessels representing propellant tanks (Symons et al., 1968; Symons and Nussle, 1969) including non-zero low gravity (Spuckler, 1972). Note that the droplet versus geyser question does not limit tank filling rates; subsequent research explored a solution or two for how to fill a tank rapidly without geysering (Abdalla and Andracchio, 1968; Labus et al., 1972). In fluids research, the droplet versus geyser question remains relevant, e.g., recent creative drop tower experiments 


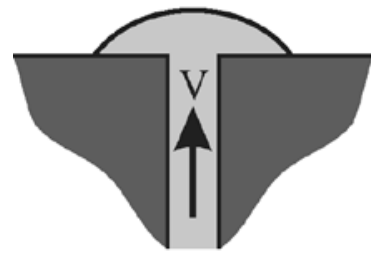

Droplet

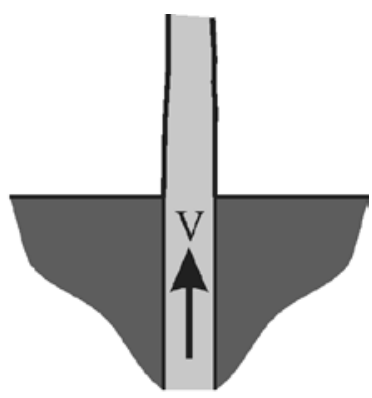

Geyser

Figure 1. Droplet and geyser geometries - light gray is the liquid, dark gray is the solid. White background is the air. Sharp orifice rims are shown here; see below for the bluntness types examined in the present research.

depend on the existence of a geyser formation at the exit of a miniature nozzle (Wollman et al., 2016). Many drop tower and flight experiments depend on injecting a volume of liquid that remains a droplet and wets the wall around an orifice.

The earlier drop tower experiments examined the flow of a liquid injected out of an orifice with what is described as a "sharp" rim. Yet there must have been some finite, albeit small, bluntness of some form at the rim of the orifice as it is not possible to manufacture a hole, especially in acrylic, with a mathematically sharp rim. Just how sharp a hole could be considered sharp and
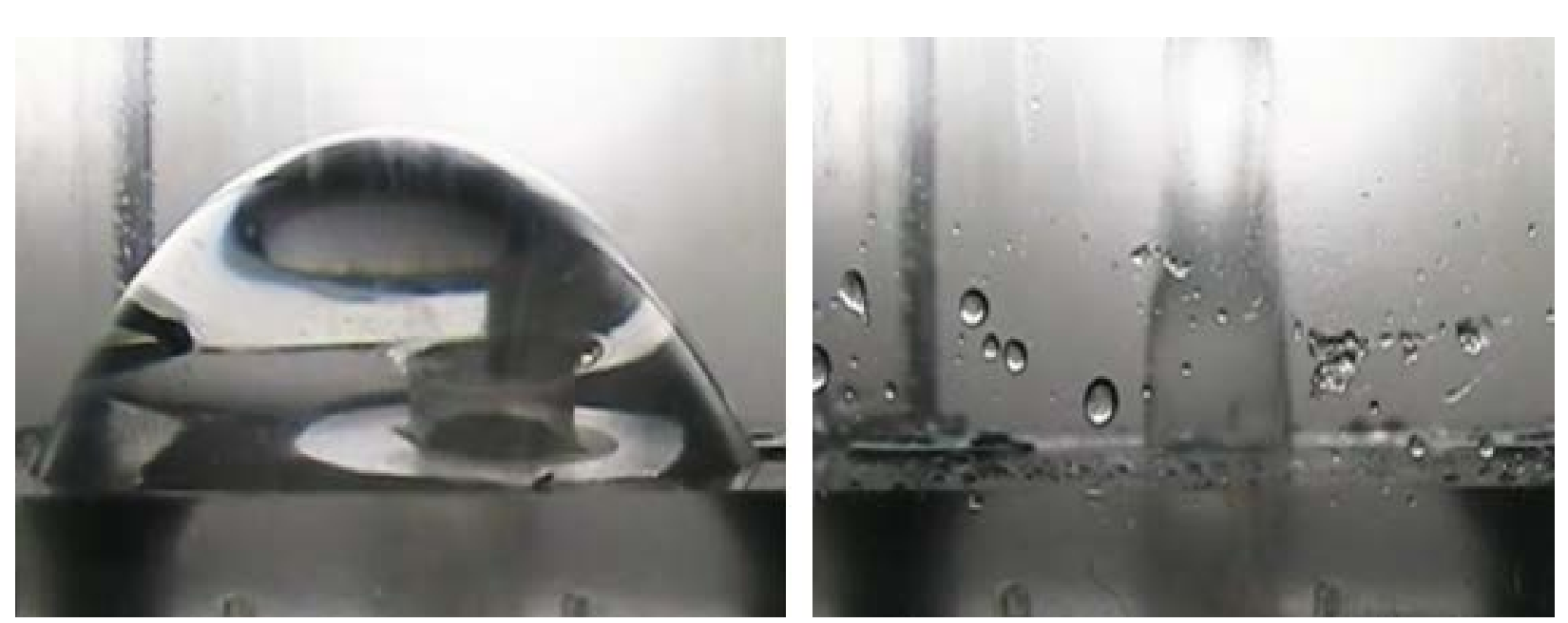

Figure 2. Droplet (left) and geyser (right) images from flight test of $0.48 \mathrm{~cm}$ diameter orifices (the two images are presented at the same scale). The small droplets in the foreground of the right image are residues of a test during a previous parabola and are in the fields of view but are not interacting with the hole or the jet.

what effect larger bluntness on the rim would have on the division between droplet and geyser formation (without regard to specific applications) are the focus of the experiments in this work.

\section{MATERIALS AND METHODS}

The relative influence of liquid momentum and capillary pressure difference across the free surface, such as in the droplet sketch and image of Figures 1 and 2, respectively, is quantified by a Weber number,

$$
W e=\frac{\rho V^{2} R}{\sigma}
$$

where the liquid density is $\rho$ (gas density is assumed negligible), the mean velocity of the liquid in the hole is $V$, orifice radius is $R$, and surface tension is $\sigma$. Deionized water is the test liquid, with ambient cabin pressure approximately the atmospheric pressure at 2,400 m altitude (75 $\mathrm{kPa}$ ) and the ambient temperature varied between 17 and $22^{\circ} \mathrm{C}$. Weber number can be viewed as the ratio of the dynamic pressure of the liquid flow to the capillary pressure difference for a hemisphere of radius equal to the orifice radius, with a factor of 4 dropped from the denominator. A spherical cap is the zero- $g$ solution for a static liquid on the rim of the orifice, and the maximum capillary pressure difference is at minimum radius for the spherical cap, which is equal to the radius of the hole. 
When the Weber number is large, the flow is dominated by liquid momentum, likely resulting in a geyser. When the Weber number is small, capillary effects dominate, and should cause a droplet to form on the wall. The question addressed in earlier work and this one is: what value of the Weber number separates the two behaviors?

To test the behavior of injected liquid through orifices of differing geometries on the rim, as shown in Figure 3, experimental apparatus was designed for parabolic aircraft flight testing. Three types of rims on the orifices are explored-sharp, chamfered, and rounded. "Sharp" cannot be interpreted literally. In this case is the label refers to rim bluntness as fine as can be machined on the transparent acrylic plates. The sharp orifice for which results are reported here has a rounding with a radius of no more than $0.04 \mathrm{~cm}$. The small orifices have diameter of 0.48 $\mathrm{cm}$ and large orifices are $0.64 \mathrm{~cm}$ diameter. Chamfer on the small and large orifices enlarges the holes to $0.70 \mathrm{~cm}$ and $0.95 \mathrm{~cm}$ diameter, respectively, at the surface of the plate. The rounded rim on the large hole is a radius of approximately $0.16 \mathrm{~cm}$.

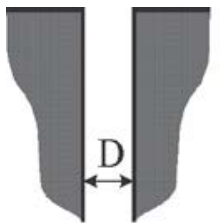

Sharp

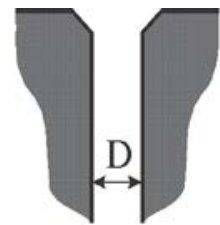

Chamfered

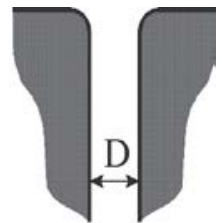

Rounded
Figure 3. Injection hole edge conditions. In this geometry, the liquid flow, when present, was upwards from the hole.

Two flights of thirty parabolas each in January, 2009 were provided by the (now defunct) NASA Reduced Gravity Student Flight Opportunity Program. Each day tested three orifices in parallel with various controlled flow rates of water. Three video camcorders (640 x 480 resolution, $30 \mathrm{fps}$ ) recorded the water as it exited the orifice. One camera failed during the flights, and thus, there are data from only four orifices analyzed in this paper. A wide range of Weber numbers were created during the first flight and results permitted adjustment of the testing plan for the second day. On the second day, the range of Weber numbers were reduced so that more experiments were conducted closer to the values of Weber numbers dividing the droplet from the geyser results.

The major components of the experiment apparatus are sketched in Figure 4. Operation for each parabola began when the nominally weightless period of the flight started and the stepper motor actuated the three pistons that pushed additional water into the plenums under the orifices causing water to flow through and out of the orifices for several seconds. Motor control was calibrated before flight to produce the desired water flow speeds through the orifices. When the viewing window is occluded by droplets, the plunger is used to wipe the window clean without breaking the double containment of the liquid. The face of the plate is, in general, partially wetted because it is found in flight that it is impractical to actuate the plunger before every parabola. A testing plan that scheduled the desired flow velocities was prepared and followed for each flight.

The video data were analyzed after the flights. For each parabola, the state of the injected fluid was assessed as a droplet, a geyser, or an indeterminate case. An indeterminate result included situations like a wall-bound drop with a satellite drop ejected out the end of the main drop, a droplet shearing off to the side, or similar, which are caused by the g-jitter of the flight.

\section{RESULTS}

All droplet, geyser, and indeterminate results from the four orifices with video records are presented versus their respective Weber number in Figure 6. Uncertainty in the Weber number is primarily from uncertainty in the surface tension of the water in the testing environment several hours after being loaded into the experiment. As a result, uncertainty in Weber number is estimated to be approximately \pm 0.08 . Density of $1 \mathrm{~g} \mathrm{~cm}^{-3}$, surface tension of 70 dyne $\mathrm{cm}^{-1}$, and the orifice radius are used in computing the Weber numbers. Uncertainty in classification, which is represented in the vertical direction in Figure 6, is absent because of the use of the indeterminate classification for collecting all of the uncertain results. Also shown in Figure 6 is a vertical dotted line for the previous result of $W e=1.3$ (Symons et al., 1968; Symons, 1970). 


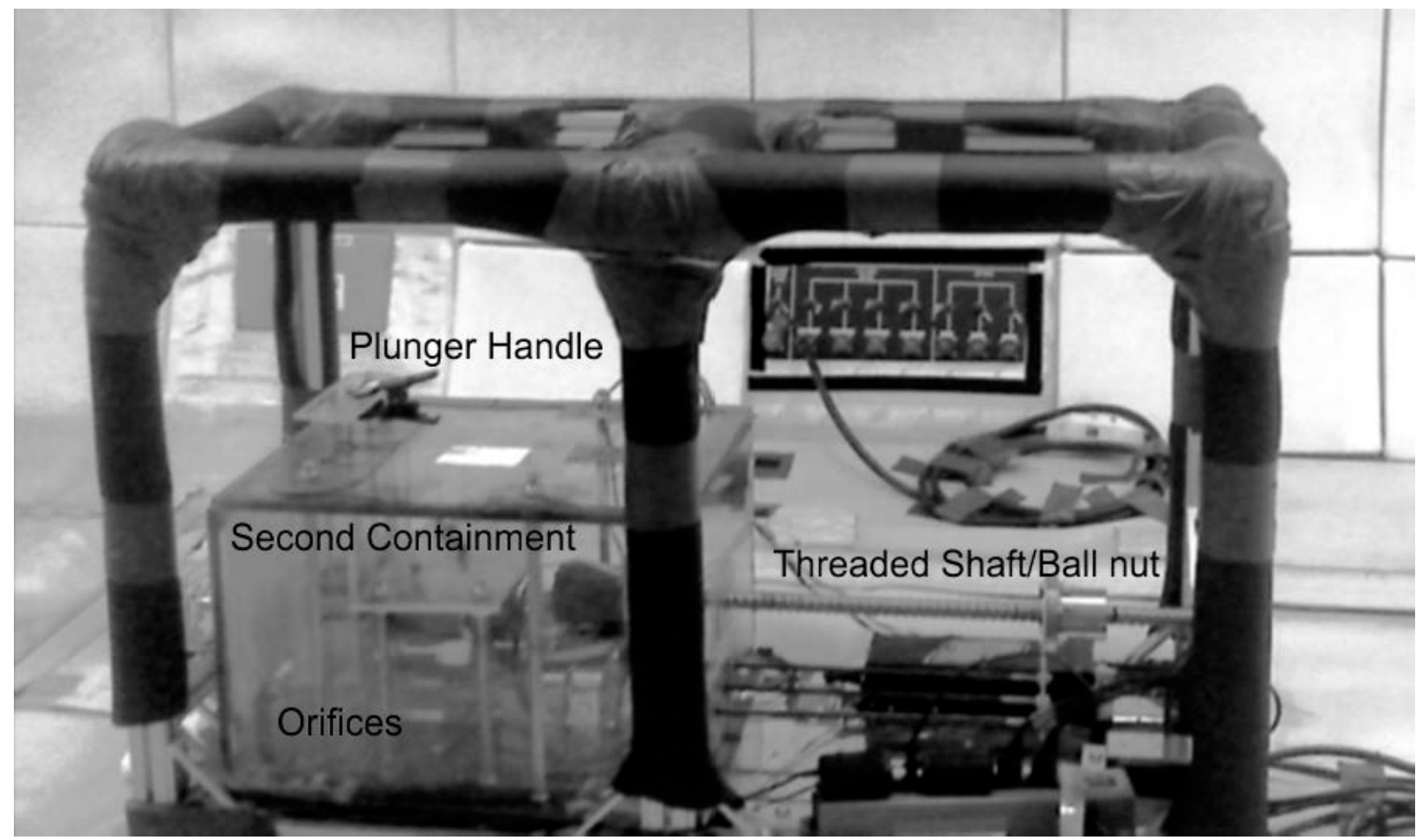

Figure 4. Photograph of experiment bolted to the floor on board the 727 of the Zero-G Corporation. The transparent box in the left half of the frame is the second level of containment around the test sections.

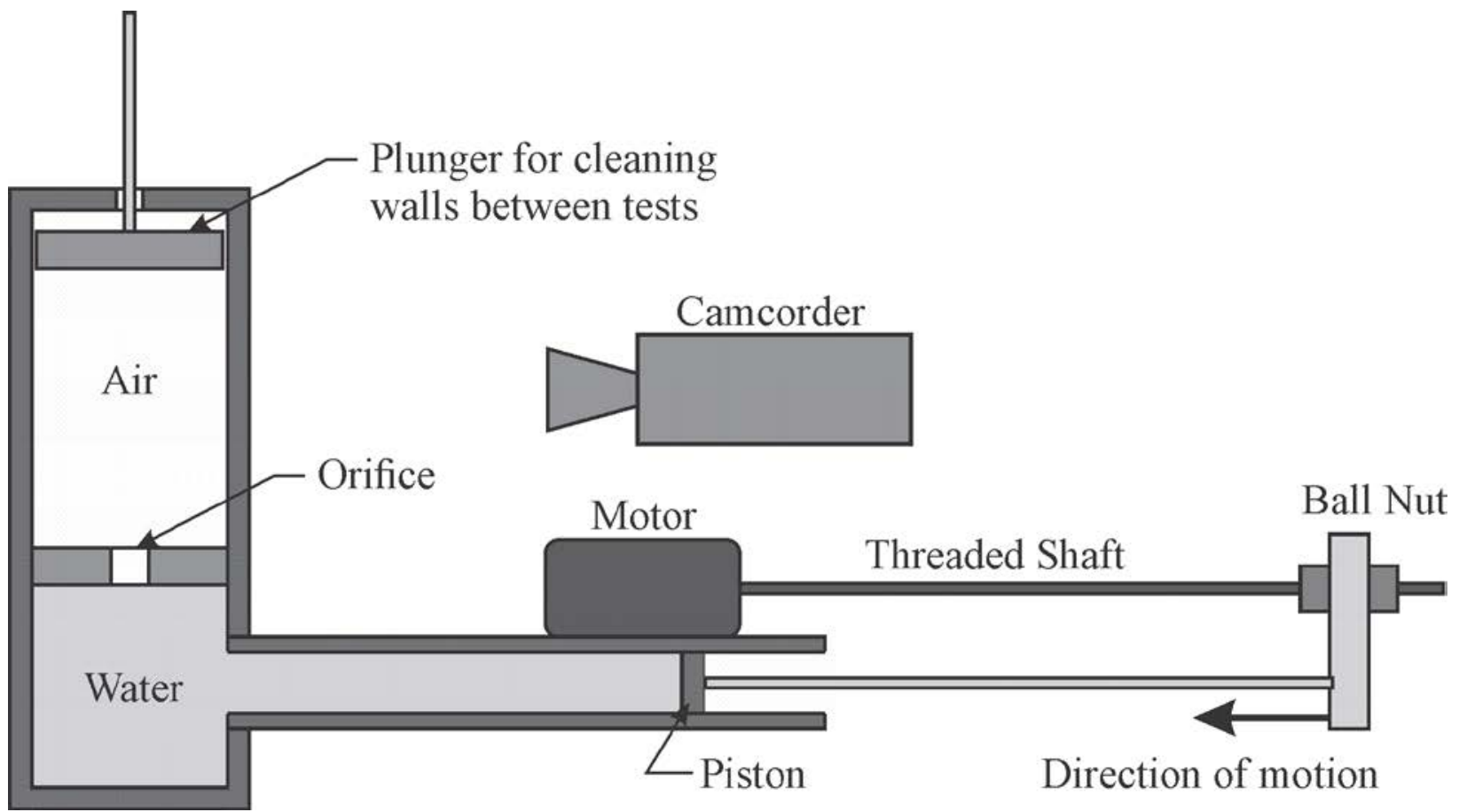

Figure 5. Experiment components, initial condition. One fluid system is shown in this view; there are two additional systems, identical except for the orifice size or rim shape, behind this system. 


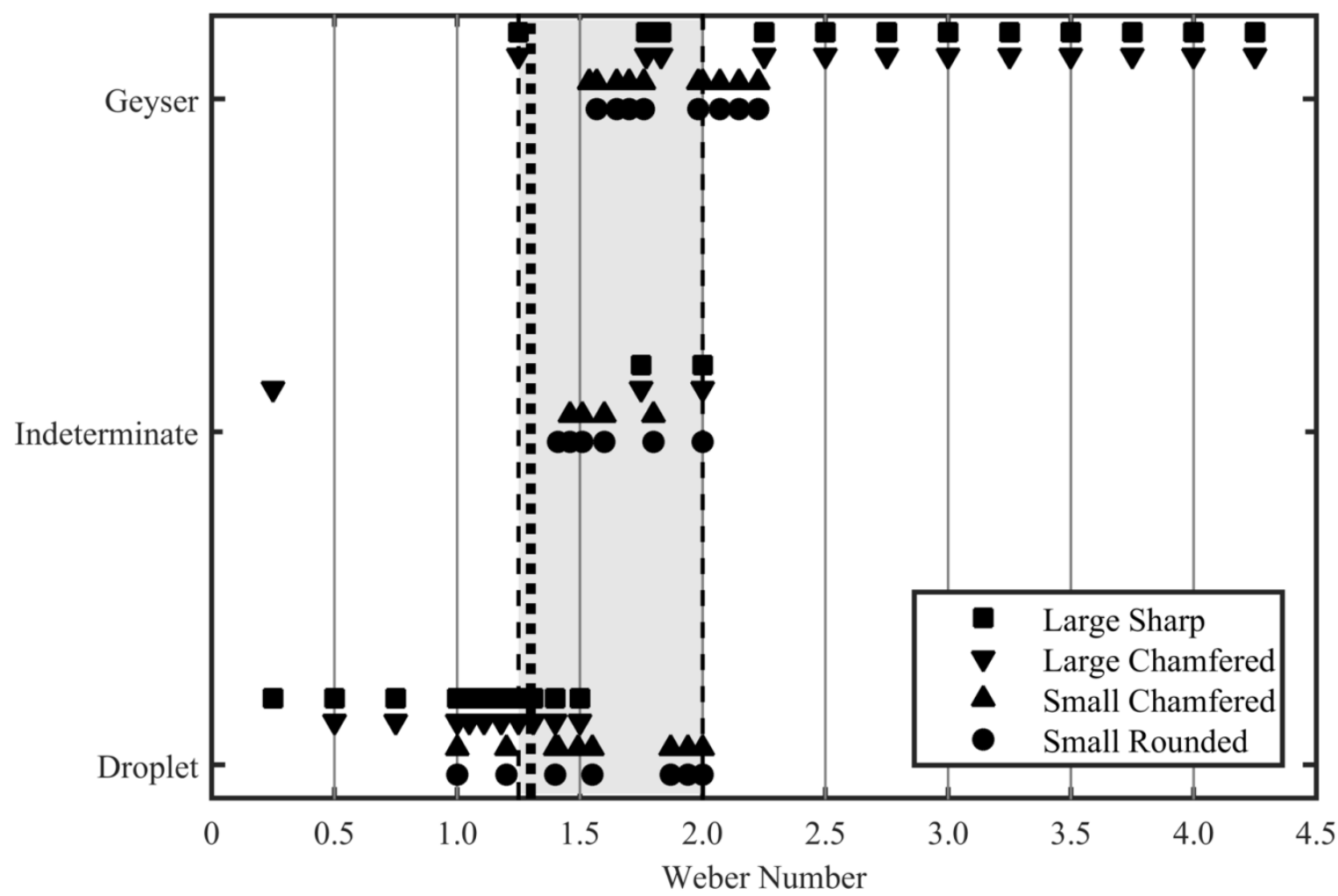

Figure 6. All of the data from four holes over two flights.

\section{DISCUSSION}

The maximum Weber number for dependable droplet formation is shown to be 1.25 . This is consistent with the previous result of 1.3 , given the uncertainty in Weber number. The minimum Weber number for dependable geyser formation is 2.0. Intermediate values of Weber number produce all three classes of results, for all four orifices. This may be the effect of g-jitter of the aircraft, a residual droplet from the preceding parabola, or other phenomena. This indeterminate condition in $1.25<W e<2$ is not inconsistent with the earlier drop tower work in which conditions are described as "unstable" above $W e=1.3$.

The indeterminate result at $W e=0.25$ stands out as unique. This case could not be classified completely as a droplet. $G$-jitter of the flight or a sticky spot for the piston driving this flow may have influenced this result. Many things can affect the droplet formation event. For example, it has even been shown that a reduction in draining rate can cause ejection of droplets (Symons, 1974).

Note that the chamfered and rounded rims are found to have no clear impact on the Weber numbers below which the droplet is formed or above which the geyser is formed. This intolerance of the results to condition of the rim of the orifice does simplify design and fabrication requirements for propellant tanks and fluids experiments alike, absent increasing importance of other phenomena.

It appears that the determination of the Weber number separating droplet and geyser production from parabolic flight experiments is found to be less precise than in experiments in drop towers. The more quiescent $g$-environment and the opportunity to clean the apparatus before each drop are the likely causes of lower ambiguity. This ambiguity may be of little consequence because in designing either a fluids experiment or a propellant transfer system, one would design to 
create a Weber number a safe distance away from the transition Weber number range.

Note that only a drop tower with the ability to drop frequently each day can produce data as rapidly as parabolic flight testing, such as would be beneficial for investigating larger chamfer sizes and rounding radii on droplet and geyser formation. In spite of the increased uncertainty compared to drop tower testing, the data in this paper from the investigation of different orifice rim geometries are new.

\section{ACKNOWLEDGEMENTS}

This experiment was enabled by the NASA Reduced Gravity Student Flight Opportunity Program and supported by the School of Aeronautics and Astronautics at Purdue University and the Indiana Space Grant Consortium. The authors express their gratitude to the above entities for the opportunity and support. Tom Brennan, Curt Freeman, Zarinah Blockton, Vimit Gandhi, and Nixon Lange plus the authors comprised the team that proposed, designed, fabricated, and operated the experiment.

\section{REFERENCES}

Abdalla KL, Andracchio CR (1968) An Experimental Study of Liquid Flow Into a Baffled Spherical Tank During Weightlessness. Washington, DC: National Aeronuatics and Space Administration, NASA-TM-X-1526

Labus TL, Aydelott JC, Andracchio CR (1972) Effect of Baffles on Inflow Patterns in Spherical Containers During
Weightlessness. Washington, DC: National Aeronuatics and Space Administration, NASA-TM-X-2670

Spuckler CM (1972) Liquid Infow to Initially Empty Cylindrical Tanks in Low Gravity. Washington, DC: National Aeronuatics and Space Administration, NASA-TM-X2613

Symons EP, Nussle RC, Abdalla KL (1968) Liquid Inflow to Initially Empty, Hemispherical Ended Cylinders During Weightlessness. Washington, DC: National Aeronuatics and Space Administration, NASA-TN-D-4628

Symons EP, Nussle RC (1969) Observations of Interface Behavior During Inflow to an Elliptical Ended Cylinder in Weightlessness. Washington, DC: National Aeronuatics and Space Administration, NASA-TM-X-1719

Symons EP (1970) Interface Stability During Liquid Inflow to Initially Empty Hemispherical Ended Cylinders in Weightlessness. Washington, DC: National Aeronuatics and Space Administration, NASA-TM-X-2003

Symons EP (1974) Effect of Throttling on Interface Behavior and Liquid Residuals in Weightlessnesss. Washington, DC: National Aeronuatics and Space Administration, NASA-TM-X-3034

Wollman A, Weislogel MM, Wiles B, Pettit D, Snyder T (2016) More investigations in capillary fluidics using a drop tower. Experiments in Fluids 57: 57. 